\title{
An Object-Based Approach to Map Human Hand Synergies onto Robotic Hands with Dissimilar Kinematics
}

\author{
G. Gioioso, G. Salvietti, M. Malvezzi and D. Prattichizzo
}

\begin{abstract}
Robotic hands differ in kinematics, dynamics, programming, control and sensing frameworks. Borrowing the terminology from software engineering, there is a need for middleware solutions to control the robotic hands independently from their specific structure, and focusing only on the task.

Results in neuroscience concerning the synergistic organization of the human hand, are the theoretical foundation of this work, which focuses on the problem of mapping human hand synergies on robotic hands with dissimilar kinematic structures.

The proposed mapping is based on the use of a virtual ellipsoid and it is mediated by a model of an anthropomorphic robotic hand able to capture the idea of synergies in human hands. This approach has been tested in two different robotic hands with an anthropomorphic and non-anthropomorphic kinematic structure.
\end{abstract}

\section{INTRODUCTION}

Among the different solutions presented in the literature, bio-inspired control of the robotic hands seems to be one of the more promising approach [1], [2]. A deeper understanding on how the brain exploit the high redundancy of the human hands could represent a key issue in the next generation of control algorithms.

In the last two decades, neuroscientists have studied the organization of the human hand in grasping and manipulation tasks using accurate hand tracking systems [3]. In particular, some studies demonstrated that, notwithstanding the complexity of the human hand, a few variables are able to account for most of the variance in the patterns of human hands configuration and movement [4]. These conclusions were based on the results of experimental tests in which subjects were asked to perform grasping actions on a wide variety of objects. Data were recorded by means of data gloves and were analysed with principal component analysis (PCA) techniques. The results showed that the first two principal components account for most of the variability in the data, more than $80 \%$ of the variance in the hand postures. In this context the principal components were referred to synergies, to capture the concept that, in the sensorimotor system of the human hand, combined actions are favoured over individual component actions, with advantages in terms

This work was partially supported by the European Commission with the Collaborative Project no. 248587, "THE Hand Embodied", within the FP7-ICT- 2009-4-2-1 program "Cognitive Systems and Robotics" and the Collaborative EU-Project "Hands.dvi" in the context of ECHORD (European Clearing House for Open Robotics Development).

G. Gioioso, G. Salvietti and M. Malvezzi are with Department of Information Engineering, University of Siena, 53100 Siena, Italy [ gioioso, salviettigio, malvezzi] ddii.unisi.it

D. Prattichizzo is with Department of Information Engineering, University of Siena and Italian Institute of Technology, Genova, Italy [prattichizzo] ddii.unisi.it

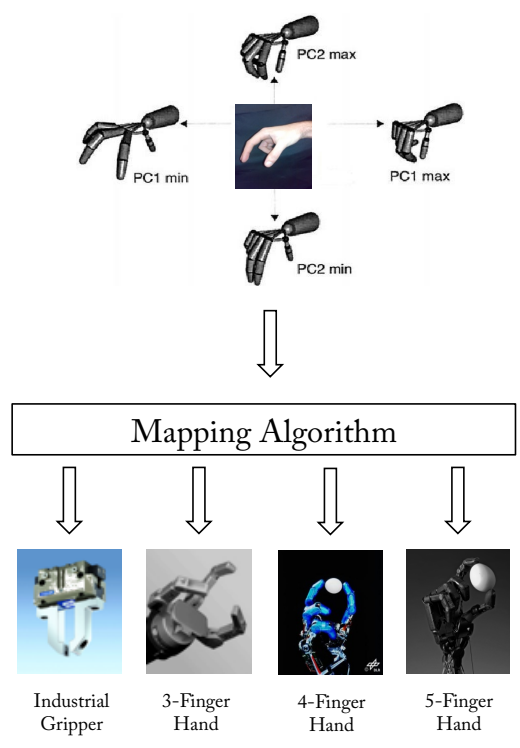

Fig. 1. Mapping between human synergies and robotic hands.

of simplification and efficiency of the overall system.

This reduction of Degrees of Freedom (DoFs) can be used to decrease the complexity of control algorithm for robotic hands. Intuitively, this approach can be applied to control robotics hands with an anthropomorphic structure that closely copy the structure of human hands. The development of a unified framework for programming and controlling robotic hands with dissimilar kinematic may extend the use of these devices in many areas.

This kind of generalization can be achieved through a mapping algorithm able to reproduce the movements due to a synergistic control of a paradigmatic model of the human hand onto the robotic hands. This mapping function focuses on the task space avoiding to consider the peculiar kinematics of the robotic hands. In Fig. 1 the idea of the proposed approach is pictorially represented.

In this paper we present a method to map human synergies onto robotic hands by using a virtual object. The target is to reproduce deformations and movements exerted by a paradigmatic human-like hand on a virtual ellipsoid computed as the minimum volume ellipsoid containing opportune reference points that lie on the hand structure. This allows us to work directly on the task space avoiding a specific projection between different kinematics. The paper generalizes the procedure described in [5], introducing the possibility to model different types of virtual object shapes 
and deformations.

The paper is organized as follows. In Section II a review of the literature is presented. Section III summarizes the main results concerning grasp properties in synergy actuated hands and describes the mapping method in detail. In Section IV some simulations are shown to confirm the proposed approach effectiveness, while the advantages and drawbacks of the proposed method together with conclusion and future work are outlined at the end.

\section{RELATED RESEARCH WORK}

Mapping algorithms are frequently used in several applications and in particular in tele-manipulation and learning by demonstration. The typical approach adopted to telemanipulate robotic hands is to use datagloves to collect human hand motion data. In [6], for example, a DLR Hand is controlled through a CyberGlove, while in [7] a master-slave teleoperation system is developed to evaluate the effectiveness of tele-presence in tele-robotics applications.

In learning by demonstration applications, data collected by human actions are not directly used to control robotic hands, but to improve the grasping performance by teaching to the robot the correct posture to obtain stable grasps. In [8], authors evaluated human grasps during an arm transportation sequence in order to learn and represent grasp strategies for different robotic hands, while in [9] a programming by demonstration system for grasp recognition in manipulation tasks and robot pre-grasp planning is developed.

If the considered robotic hand is strongly anthropomorphic, the data collect from the humans can be used straightforward in both tele-manipulation and learning by demonstration applications. When robotic hands have different kinematics, mapping strategies have to be taken into account. There are three main approaches in literature to deal with this problem: joint to joint mapping, Cartesian Space or fingertip mapping and pose mapping.

The aim of the first approach is to make the poses of human and robotic hands look similar. Ciocarlie and Allen, for instance, used this method to take advantage of human hand synergies in robotic grasp pre-shaping [10]. They tested four different robotic hands with this procedure: the human hand joint values were directly mapped into the joints of the anthropomorphic hands, while some empirical solutions were adopted with the non-anthropomorphic hands. This represents the simplest way to map movements between hands. Anyway, joint to joint method has to be redefined according to the kinematic characteristics of the hands making difficult a generalization of the control strategy considering also that the performance notably decrease with non-anthropomorphic structures.

Cartesian Space mappings focus on the geometric relations between the two workspaces. This solution is suitable for representing the fingertip positions and it is a natural approach when, for example, precision grasps are considered. In [11] a point-to-point mapping algorithm is presented for a multi-fingered telemanipulation system where fingertip motion of the human hand is reproduced with a three-finger robotic gripper. In [12] authors used a virtual finger solution to map movements of the human hand onto a four-fingered robotic hand. However, these solutions do not guarantee a correct mapping in terms of forces and movements exerted by the robotic hand on a grasped object.

The pose mapping can be considered as a particular way of indirect joint angle mapping. The basic idea of the pose mapping is trying to establish a correlation between human hand poses and robotic hand poses. For example, Pao and Speeter [13] developed an algorithm that tries to translate human hand poses to corresponding robotic hand positions, without loss of functional information and without the overhead of kinematic calculations. In [14] neural networks were used to learn the hand grasping postures. Anyway, the proposed solutions can produce unpredictable motions of the robot hand, and thus in our opinion are only exploitable in those cases where basic grasp postures are required.

Besides the above mentioned methods, combinations of them and some original solutions, as the method proposed in [15], are also present in literature. In [16] a virtual object-based mapping is proposed. The object based scheme assumes that a virtual circle is held between the user's thumb and index finger. Important parameters of the virtual object (the size, position and orientation) are scaled independently and non-linearly to create a transformed virtual object in the robotic hand workspace. This modified virtual object is then used to compute the robotic fingertip locations that in this case is a simple two-fingers, four-DoFs gripper.

A 3D extension of the last method is presented in [17]. Even if this extension allows to analyse more cases, this method is still not enough general for our purposes. In particular, it is constrained by the kinematics of the master and slave hand, the number of contact points (three) and their locations (the fingertips) which have to be the same for both the hands. Then, it can be used only for a given pair of human and robotic hands and for precision grasp operations.

The approach proposed in this paper is inspired by the last two mentioned methods. The main contributions of our work with respect to the methods proposed in [16], [17] are here summarized: our approach generalizes the mapping to a generic number of contact points that can be different in the human and robotic hands and there are no constraints on positions of contact points on the master and on the slave hand. Moreover, to the best of our knowledge, none of the presented papers deal with mapping synergies of the human hand onto robotic hands, defined as a reduced dimension of the configuration space.

\section{OBJECT-BASED MAPPING}

\section{A. Synergy background}

In this section the main equations necessary to study hands controlled by synergies from a kinematic point of view are introduced. A more detailed presentation of the problem is described in [18], further details on grasp theory can be found in [19], [20].

Let us consider a hand grasping an object with no distinction between human or robotic. The hand can be represented 
as a mechanical interface composed of a series of kinematic chains, the fingers, that share a common base, the palm. The fingers and/or the palm is connected to the manipulated object by some contact patches, usually represented as points (even though, due to the finger and object compliance, they are extended to finite areas).

Once the contact points on the hand are defined, the conventional robotic analysis tools allow to find a relationship between their locations with respect to hand palm and the finger joint variable (direct kinematics). This relationship can be differentiated with respect to time, giving the differential kinematic equation that relates the contact point velocities $\dot{p} \in \mathfrak{R}^{n_{c}}$ to the joint velocities $\dot{q} \in \mathfrak{R}^{n_{q}}$

$$
\dot{p}=J \dot{q}
$$

where $J$ is the hand Jacobian $J \in \Re^{n_{c} \times n_{q}}, n_{q}$ is the number of actuated joints and $n_{c}$ is the number of contact variables (components of the contact point twists) constrained by the contact model.

The transpose of hand Jacobian relates the grasping contact forces $\lambda \in \mathfrak{R}^{n_{c}}$ to the hand joint torques $\tau \in \mathfrak{R}^{n_{q}}$

$$
\tau=J^{\mathrm{T}} \lambda
$$

Contact point twist components on the grasped object are related to the object reference frame twist $v^{o}=\left[\begin{array}{ll}\dot{o}^{\mathrm{T}} & \omega^{\mathrm{T}}\end{array}\right]^{\mathrm{T}}$ by the following relationship

$$
\dot{p}=G^{\mathrm{T}} v^{o} .
$$

where $G^{\mathrm{T}}$ denotes the grasp matrix transpose, $G \in \mathfrak{R}^{6 \times n_{c}}$. The Grasp matrix relates the grasping contact forces $\lambda$ to the object external wrench $w \in \mathfrak{R}^{6}$

$$
w=G \lambda
$$

For further details on hand Jacobian and Grasp matrices definition and computation, the reader is referred to [20].

According to a model inspired by human hand synergies, we suppose that the hand is actuated using a number of inputs whose dimension is lower than the number of hand joints. These inputs are then collected in a vector $z \in \mathfrak{N}^{n_{z}}$ that parametrize the hand motions along the synergies. In this paper we define the postural synergies as a joint displacement aggregation corresponding to a reduced dimension representation of hand movements, according to a compliant model of joint torques.

In other terms, the reference vector $q_{r e f} \in \mathfrak{R}^{n_{q}}$ for joint variables is a linear combination of postural synergies $z \in \mathfrak{R}^{n_{z}}$ with $n_{z} \leq n_{q}$

$$
q_{r e f}=f_{z}(z)
$$

This approach differs from other works where the synergies are considered perfectly stiff and the actual joint variables are a linear combination of synergies [21], [22]. In a compliant actuation model, the torques applied by the joint motors is proportional to the difference between the reference and the actual joint displacement

$$
\tau=K_{q}\left(q_{r e f}-q\right)
$$

where $K_{q}$ is the joint stiffness matrix. It represents joint actuator stiffness, given by the joint position control static gain, and the structural compliance, due to the mechanical deformation of hand elements (joints, drive cables, links, etc.). The problem of computing contact force distribution and object movements that can be controlled acting on synergies has been studied in [18].

The relationship in eq. (4) can be differentiated with respect to time, obtaining

$$
\dot{q}_{r e f}=S \dot{z}
$$

where the synergy matrix $S \in \mathfrak{R}^{n_{q} \times n_{z}}$, is defined as

$$
S=\frac{\partial f_{z}}{\partial z}
$$

Matrix $S$ columns describe the shapes, or directions, of each synergy in the joint velocity space. It is worth noting that, if the relationship defined in eq. (4) is not linear, synergy matrix $S$ is not constant, but depends on synergy value $z$.

\section{B. Mapping Algorithm}

The target of this work is to determine a method to map a set of synergies defined on a reference human hand onto a generic robotic hand. A model of the human hand is thus defined. We refer to this model as paradigmatic hand. The paradigmatic hand is a kinematic and dynamic model inspired by the human hand that does not closely copy the kinematical and dynamical properties of the human hand but rather represents a trade-off between the complexity of the human hand model accounting for the synergistic organization of the sensorimotor system and the simplicity, and accessibility, of the models of robotic hands available on the market. A detailed kinematic analysis of the paradigmatic hand with postural synergies is reported in [23].

Let the paradigmatic hand be described by the joint variable vector $q_{h} \in \mathfrak{R}^{n_{q_{h}}}$ and assume that the subspace of all configurations can be represented by a lower dimensional input vector $z \in \mathfrak{R}^{n_{z}}$ (with $n_{z} \leq n_{q_{h}}$ ) which parametrizes the motion of the joint variables along the synergies $q_{h}=S_{h} z$ being $S_{h} \in \mathfrak{R}^{n_{q_{h}} \times n_{z}}$ the synergy matrix. In terms of velocities one gets

$$
\dot{q}_{h}=S_{h} \dot{z} .
$$

The ultimate goal of this work is to find a way of controlling the joint variables $\dot{q}_{r} \in \mathfrak{R}^{n_{q_{r}}}$ of the robotic hand in a synergistic way using the vector of synergies $z$ of the paradigmatic hand. In other terms we want to design a map $S_{r}$ to steer the robotic joint variables as follows

$$
\dot{q}_{r}=S_{r} \dot{z}
$$

where map $S_{r}$ depends on synergy matrix $S_{h}$ and other variables as explained in the following.

Remark 1: Actually, according to the compliant model previously summarized, and according to eq. (6), in eq. (7) and (8) we should indicate the reference hand joint values $\dot{q}_{h_{r e f}}$ and $\dot{q}_{r_{r e f}}$ respectively. It is worth noting that the mapping procedure explained in this paper considers a virtual grasp, i.e. a grasp in which no real contact forces are present, 
and then, according to eq. (2) and (5), there is no difference between the reference and the actual hand configuration. However, the mapping procedure can be applied even when a real grasp is considered: in this case it will provide the reference value of joint variables, that will differ from the actual one, and the difference will depend both on the contact force magnitude and on the system compliance. In the following equations, for the sake of simplicity, we will not use the subscript ref to indicate the reference joint values. $\square$

In this work, we propose a method of projecting synergies from paradigmatic to robotic hands which explicitly takes into account the task space. One of the main advantages of designing a mapping strategy in the task space is that results can be used for robotic hands with very dissimilar kinematics. The idea is to replicate the task performed with the paradigmatic hand using the robotic hand with projected synergies.

The mapping is defined assuming that both the paradigmatic and the robotic hands are in given configurations $q_{0 h}$ and $q_{0 r}$.

Remark 2: Note that the mapping procedure can be applied for any pair of reference configurations $q_{0 h}$ and $q_{0 r}$, i.e. paradigmatic and robotic hand configurations can be set independently. However, the choice of very dissimilar initial configuration may lead to hand trajectory that appears very different in the configuration space, although they produce, on the virtual object, the same displacement and the same deformation. Very dissimilar initial configuration may also lead to other types of problems, for example one of the hands may reach its joint limits or singular configurations while the other could further move.

Given the configuration $q_{0 h}$, a set of reference points $p_{h}=\left[p_{1 h}^{\mathrm{T}}, \cdots, p_{i h}^{\mathrm{T}}, \cdots\right]^{\mathrm{T}}$ are chosen on the paradigmatic hand. In this work we considered the fingertip points as reference points. However, the algorithm can be applied also choosing other reference points, for example on the intermediate phalanges or in the hand palm, and furthermore the number of reference points is not a-priori fixed. Adding other reference point can be useful when a power grasp is taken into account. The virtual ellipsoid is then computed as the minimum volume ellipsoid containing the reference points in $p_{h}$ (Fig. 2 ). Note that in general reference points do not lie on the ellipsoid surface. Let us parametrize the virtual ellipsoid by its center $o_{h}$ and by the lengths $s_{j h}(j=1,2,3)$ of its semi-axes. The motion of the hand due to synergies activation could be described using a large set of parameters. In this paper we simplify the problem assuming a rigid-body motion, defined by the linear and angular velocities of the ellipsoid center $\dot{o}_{h}$ and $\omega_{h}$ respectively, and a non-rigid strain represented by the variations of the semi-axes. Let $\dot{s}_{j}$ be the derivative of the $j$-th semi-axis length.

Although the virtual ellipsoid does not represent an object grasped by the paradigmatic hand, it can be easily shown that with a suitable model of joint compliance and contact compliance, the rigid-body motion of the virtual ellipsoid corresponds to the motion of a grasped ellipsoidal object

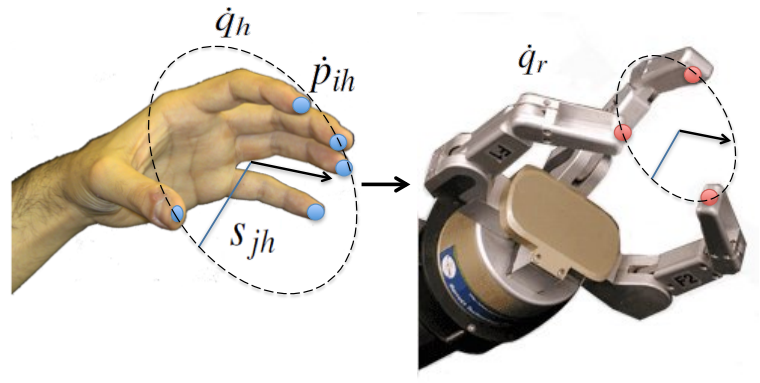

Fig. 2. Mapping synergies from the human (paradigmatic) to the robotic hand: the fingertip positions of the paradigmatic hand allows to define the virtual ellipsoid. Activating the human hand synergies, the ellipsoid is moved and strained; the same motion and strain is imposed to the virtual ellipsoid defined for the robotic hand.

and that the non-rigid motion accounts for the normal components of the contact forces for a ellipsoidal object grasp. By representing the motion of the hand through the virtual object, the motion of the generic reference point $p_{i h}$ can be expressed as

$$
\dot{p}_{i h}=\dot{o}_{h}+\omega_{h} \times\left(p_{i h}-o_{h}\right)+\sum_{j=1}^{3} \dot{s}_{j h}\left[\left(p_{i h}-o_{h}\right)^{\mathrm{T}} \hat{s}_{j h}\right] \hat{s}_{j h},
$$

where $\hat{s}_{j h}$ represent the versor corresponding to the ellipsoid semi-axes.

Grouping all the reference point motions one gets

$$
\dot{p}_{h}=A_{h}\left[\begin{array}{c}
\dot{o}_{h} \\
\omega_{h} \\
\dot{s}_{1 h} \\
\dot{s}_{2 h} \\
\dot{s}_{3 h}
\end{array}\right],
$$

where matrix $A_{h} \in \Re^{n_{c h} \times 9}$ is defined as follows

$$
A_{h}=\left[\begin{array}{cccc}
I & -S\left(p_{1 h}-o_{h}\right) & {\left[\left(p_{1 h}-o_{h}\right)^{\mathrm{T}} \hat{s}_{1 h}\right] \hat{s}_{1 h}} & \ldots \\
\ldots & \ldots & \ldots & \ldots \\
I & -S\left(p_{i h}-o_{h}\right) & {\left[\left(p_{i h}-o_{h}\right)^{\mathrm{T}} \hat{s}_{1 h}\right] \hat{s}_{1 h}} & \ldots \\
\ldots & \ldots & \ldots & \ldots
\end{array}\right]
$$

Matrix $A_{h}$ depends on the motion that has to be reproduced on the robotic hand and consequently it depends on the task. Considering the contact model as hard finger [20], the matrix $A_{h}$ can be seen as the Grasp matrix for the virtual object. From (1), (7) and (10) we can evaluate the virtual ellipsoid motion and deformation as a function of the synergy vector velocity $\dot{z}$ of the paradigmatic hand

$$
\left[\begin{array}{c}
\dot{o}_{h} \\
\omega_{h} \\
\dot{s}_{1 h} \\
\dot{s}_{2 h} \\
\dot{s}_{3 h}
\end{array}\right]=A_{h}^{\#} \dot{p}_{h}=A_{h}^{\#} J_{h} S_{h} \dot{z}
$$

where $A_{h}^{\#}$ denote the pseudo-inverse of matrix $A_{h}$. These motions and deformations have to be mapped onto the robotic hand. Let us consider the robotic hand in a given configuration $q_{0 r} \in \mathfrak{R}^{n_{q r}}$ with a set of selected reference point location vector $p_{r} \in \mathfrak{R}^{n_{c r}}$. Note that no hypothesis 
were imposed on the number of reference points on the paradigmatic human and robotic hands, in general we can consider $n_{c h} \neq n_{c r}$, neither on their locations, and neither on the initial configuration of the two hands. The same use of the virtual ellipsoid is applied here: find the minimum ellipsoid enclosing the reference points and indicate with $o_{r}$ its center coordinates and with $s_{j r}$ the lengths of its semi-axes.

In order to take into account the differences between the dimensions of the human and the robotic workspace, a scaling factor is introduced. This scaling factor is obtained considering two virtual spheres computed on both the human and the robotic hand as the minimum volume sphere containing reference points. The virtual object scaling factor is then defined as the ratio between the radii of the two spheres: $k_{s c}=\frac{r_{r}}{r_{h}}$. Note that the scaling factor depends on the hand dimensions, but also on their configurations.

The motion and deformation of the virtual ellipsoid generated by the paradigmatic hand are scaled and tracked by the virtual ellipsoid referred to the robotic hand:

$$
\left[\begin{array}{c}
\dot{o}_{r} \\
\omega_{r} \\
\dot{s}_{1 r} \\
\dot{s}_{2 r} \\
\dot{s}_{3 r}
\end{array}\right]=K_{c}\left[\begin{array}{c}
\dot{o}_{h} \\
\omega_{h} \\
\dot{s}_{1 h} \\
\dot{s}_{2 h} \\
\dot{s}_{3 h}
\end{array}\right]
$$

where the scale matrix $K_{c} \in \mathfrak{R}^{9 \times 9}$ is defined as:

$$
K_{c}=\left[\begin{array}{ccc}
k_{s c} I_{3,3} & 0_{3,3} & 0_{3,3} \\
0_{3,3} & I_{3,3} & 0_{3,3} \\
0_{3,3} & 0_{3,3} & I_{3,3}
\end{array}\right] .
$$

According to eq. (10) and (11), the corresponding robot reference point velocity is given by

$$
\dot{p}_{r}=A_{r}\left[\begin{array}{c}
\dot{o}_{r} \\
\omega_{r} \\
\dot{s}_{1 r} \\
\dot{s}_{2 r} \\
\dot{s}_{3 r}
\end{array}\right],
$$

where matrix $A_{r} \in \mathfrak{R}^{n_{c r} \times 9}$ is defined as follows:

$$
A_{r}=\left[\begin{array}{cccc}
I & -S\left(p_{1 r}-o_{r}\right) & {\left[\left(p_{1 r}-o_{r}\right)^{\mathrm{T}} \hat{s}_{1 r}\right] \hat{s}_{1 r}} & \cdots \\
\ldots & \cdots & \cdots & \cdots \\
I & -S\left(p_{i r}-o_{r}\right) & {\left[\left(p_{i r}-o_{r}\right)^{\mathrm{T}} \hat{s}_{1 r}\right] \hat{s}_{1 r}} & \cdots \\
\ldots & \ldots & \cdots & \cdots
\end{array}\right]
$$

Recalling eq. (12) and (13) we can express the robotic hand reference point velocities $\dot{p}_{r}$ as a function of the synergy velocities $\dot{z}$ :

$$
\dot{p}_{r}=A_{r} K_{c} A_{h}^{\#} J_{h} S_{h} \dot{z}
$$

and, considering the robot hand differential kinematics $\dot{p}_{r}=$ $J_{r} \dot{q}_{r}$, where $J_{r} \in \mathfrak{R}^{n_{c r} \times n_{q r}}$ is its Jacobian matrix, the following relationship between robot hand joint velocities and synergy velocities is defined:

$$
\dot{q}_{r}=J_{r}^{\#} A_{r} K_{c} A_{h}^{\#} J_{h} S_{h} \dot{z} .
$$

Then the synergy mapping $S_{r}$ in (8) for the robotic hand is defined as

$$
S_{r}=J_{r}^{\#} A_{r} K_{c} A_{h}^{\#} J_{h} S_{h}
$$

Note that the paradigmatic hand synergy matrix $S_{h}$ is mapped to the synergy matrix for the robotic hand $S_{r}$ through matrix $J_{r}^{\#} A_{r} S_{c} A_{h}^{\#} J_{h}$ which is function of paradigmatic and robotic hand configurations $\left(q_{0 h}\right.$ and $\left.q_{r h}\right)$ and, of location of the reference points for the paradigmatic and robotic hands ( $p_{h}$ and $p_{r}$ ).

The proposed algorithm is consequently a non-linear mapping between the paradigmatic human-like hand and the robotic hand. The obtained synergy matrix is not constant and depends on hands configurations.

\section{Simulations}

We validated the proposed approach on a modular threefingered 9 DoFs robotic hand and on a DLR-HIT II Hand model with five fingers and 15 DoFs [24]. We compared our results with the joint to joint mapping and the fingertipmapping methods [10], [11]. Other mapping methods [14], [16] were not taken into account since they can not be easily extended to kinematic structures that differ from those proposed in the relative applications.

The grasp of two different objects was considered: a sphere and a cube. The paradigmatic and robotic hand joint variables, and the contact points in the initial configurations were known. Starting from this initial given grasp, we modified the reference joint values according to the previously described methodology. Since the hand is grasping an object, by activating the synergies, both the contact forces and the grasped object position and orientation vary. The details of the relationships between input synergy and output variable values are detailed in [18]. Algorithm performances were evaluated comparing the object motion directions and grasp quality obtained controlling the robotic hands with the above mentioned algorithms [18], [23].

Grasp quality evaluation was performed using both qualitative and quantitative metrics in order to evaluate the forceclosure properties of the grasp as described in [25]. The qualitative metric returns a boolean value that shows if the obtained grasp is force-closure. The quantitative aspect of the grasp quality is expressed using a penalty function. The resulting index represents the inverse of the distance of the grasp from violating contact constraints. All details of the used indexes can be found in [25].

In the first simulation, a spherical object was considered and the reference points for the human and robotic hands were chosen on their respective fingertips.

We considered the paradigmatic hand grasping a sphere with the fingertips of the thumb, index, medium and ring fingers, while for the modular three-fingered hand and the DLR-HIT II hand we considered three and four contact points respectively. This emphasizes the independence of our method to the number of selected contact points. The paradigmatic and robot hand grasps that were analysed are shown in Fig. 3. The computed scaling factors $k_{s c}$ were 1.7 

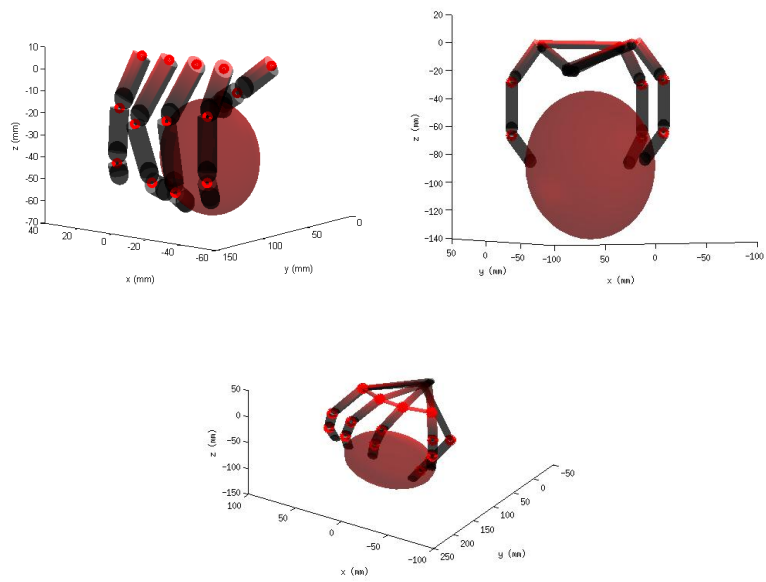

Fig. 3. The human-like hand (top-left), the modular three- finger robotic hand (top-right) and the DLR-HIT II Hand (bottom) grasping a sphere with respectively four, three and four contact points.

and 1.9 for the modular and the DLR-HIT hand respectively.

The obtained results are summarized in Table I for the DLR hand and for the modular hand, respectively. Each row corresponds to the case of controlling hands with one synergy or combinations of synergies. This analysis was carried out considering the first three synergies and their combinations.

The second column shows the grasp quality indexes for the human-like hand controlled with synergies, while the third one reports those of the robotic hand controlled with the reference joint values obtained with the proposed virtual ellipsoid mapping. The fourth and the fifth columns refer to the joint to joint mapping and to the fingertip mapping, respectively [10], [11]. The performance is expressed by means of the cost function measuring the grasp quality as described in [25]. The selected cost function basically represents a sort of distance between the contact forces and the direction normal to the contact surface, then lower values represent set of contact forces that are farther from the friction cone boundaries and then are better from the grasp stability point of view. This cost function can be evaluated only if the grasp is force-closure, empty values in the table mean that, for that method and those selected synergies, no force closure is achievable. More details on the evaluation of grasp quality measures in synergy actuated robotic hands can be found in [23].

Let us analyse, for example, results shown in Table I, relative to the DLR hand. In the paradigmatic hand (second column), force closure can be obtained by activating only the first, the first two or the first three synergies, no force closure can be obtained if we activate only the second or third synergy once. By increasing the number of synergies from one to three clearly grasp quality increases (cost function value decreases). If we consider the virtual ellipsoid mapping method described in the preceding section, we obtain better results with respect to the joint to joint and the fingertip methods. The same quality indexes were evaluated consid- ering the grasp of an object with different shape, a cube. The obtained results are summarized in Tables II for the DLR hand (the modular hand presents qualitatively similar results). By observing the obtained results, we can conclude that, concerning the grasp quality index, the virtual ellipsoid mapping, both for spherical and cubic objects gets closer to the human-like grasp behaviour in all the analysed cases.

TABLE I

GRASP QUALITY EVALUATION FOR THE SPHERICAL OBJECT.

DLR-HIT II hand

\begin{tabular}{|l|c|c|c|c|}
\hline Synergies & P & VE & JtJ & F \\
\hline Syn 1 & 0.2 & - & - & - \\
Syn 2 & - & - & - & - \\
Syn 3 & - & - & - & - \\
Syn [1-2] & 0.14 & 0.046 & - & 0.079 \\
Syn [1-3] & 0.09 & 0.037 & 0.057 & 0.049 \\
\hline
\end{tabular}

Modular hand

\begin{tabular}{|l|c|c|c|c|}
\hline Synergies & P & VE & JtJ & F \\
\hline Syn 1 & 0.2 & - & - & - \\
Syn 2 & - & - & - & - \\
Syn 3 & - & - & - & - \\
Syn [1-2] & 0.14 & 0.02 & 0.113 & - \\
Syn [1-3] & 0.09 & 0.02 & 0.020 & - \\
\hline
\end{tabular}

P: paradigmatic hand, VE: Virtual Ellipsoid, JtJ: Joint to Joint, F: Fingertip

TABLE II

GRASP QUALITY EVALUATION FOR THE CUBIC OBJECT.

DLR-HIT II hand

\begin{tabular}{|l|c|c|c|c|}
\hline Synergies & P & VE & JtJ & F \\
\hline Syn 1 & 0.3 & - & - & - \\
Syn 2 & - & - & - & - \\
Syn 3 & - & - & - & - \\
Syn [1-2] & 0.20 & 0.156 & - & - \\
Syn [1-3] & 0.14 & 0.146 & - & - \\
\hline
\end{tabular}

P: paradigmatic hand, VE: Virtual Ellipsoid, JtJ: Joint to Joint, F: Fingertip

We further evaluated the error arising from the mapping of a rigid body motion of an ellipsoidal object. The rigid body motion was obtained by applying a constant synergy rate, $i$. e. $\dot{z}=$ const . We firstly evaluated the trajectory of the virtual ellipsoid with the paradigmatic hand. We then compared this trajectory with those obtained with the DLR-HIT II hand, by applying the proposed virtual ellipsoid (VE), the joint to joint (JtJ) and the fingertip (F) mapping procedures. In the virtual ellipse algorithm, we used a scale factor $k_{s c}=1$, in order to compare the trajectories for the paradigmatic and robotic hand respectively. According to the geometric analysis presented in [18], that relates the dimensions of controllable internal contact force and rigid body motion subspaces to the number of actuated synergies, we observed that, if an object rigid body motion has to be produced, 

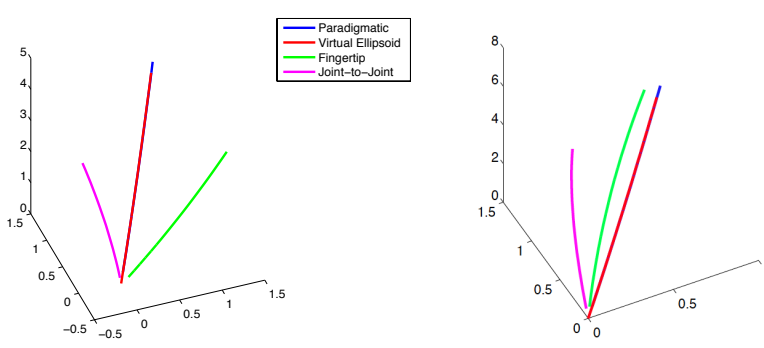

Fig. 4. Trajectories in the workspace of the center of the ellipsoidal object for the modular (left) and DLR-HIT II (right) hand. The blue lines represent the mapped motion obtained with the proposed algorithm; the red line represent the motion with the paradigmatic hand, while green and violet lines represent joint to joint and fingertip mapping respectively. Axis units are in $\mathrm{cm}$.

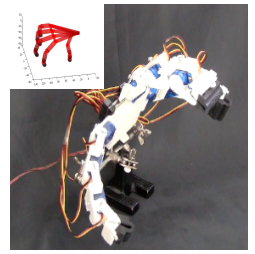

(a)

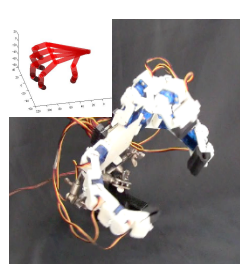

(b)

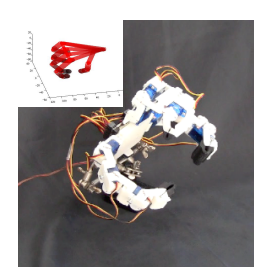

(c)
Fig. 5. The first synergy mapped onto the modular hand: (a) starting position, (b) middle position, (c) end position. In the top-left the corresponding paradigmatic hand postures.

at least four synergies have to be considered with three contact points (with a Hard Finger contact model) and seven synergies with four contact points. For the DLR-HIT II hand analysis we then considered the first seven synergies. The arising trajectories of the center of the ellipsoidal object for modular and DLR-HIT II hand are shown in Fig. 4. As it can be seen from the plots, the trajectories obtained for the robotic hand with the virtual ellipsoid procedure is very close to those obtained with the paradigmatic hand, with respect to the other mapping methods. This result is clearly due to the fact that the mapping itself is based on the replication, in the workspace, of the same rigid body motion of a virtual object.

\section{EXPERIMENTS}

The proposed mapping procedure has been validated by some experiments performed with a fully-actuated robotic hand with a modular structure. Each module $(42 \times 33 \times$ $16 \mathrm{~mm}$ ) has one DoF and it can be easily connected to the others obtaining kinematic chains that we can consider as fingers. These chains are connected to a common base that can be thought as a palm. In the proposed configuration each finger has three DoFs, thus the hand has globally nine DoFs.

The first two synergies of the paradigmatic hand, mapped on the modular robotic hand according to eq. (19), are shown in Fig. 5, 6.

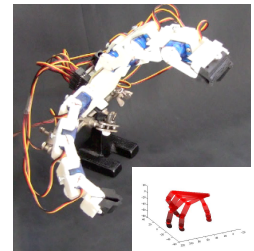

(a)

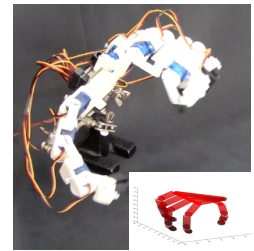

(b)

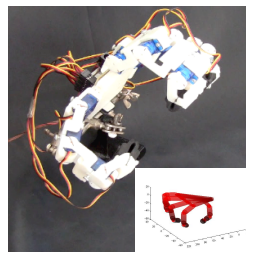

(c)
Fig. 6. The second synergy mapped onto the modular hand: (a) starting position, (b) middle position, (c) end position. In the bottom-right the corresponding paradigmatic hand postures.

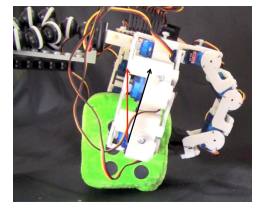

(a)

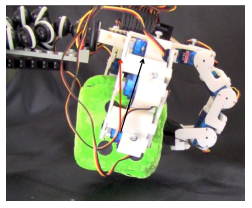

(b)

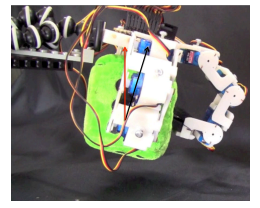

(c)
Fig. 7. Object rigid body motion obtained controlling the first four synergies: (a) starting position, (b) middle position, (c) end position. The red arrows represent the motion of the object, while the black arrows represent the movement imposed by the paradigmatic hand.

Concerning the rigid body object motion obtained controlling the robotic hand with synergies calculated according to the method described in the preceding sections and summarized in eq. (19), the results are shown in Fig. 7.

Although the used device represents a trivial example of robotic hand, the complexity and the high number to DoFs to control are, in our opinion, a possible benchmark to validate our approach. Furthermore its kinematic structure is significantly different from the paradigmatic hand one, so it could be useful to test how the proposed mapping method behaves with very dissimilar hand structures.

\section{DISCUSSION}

The proposed mapping strategy, based on mimicking behaviour of human hand synergies, could be the basis of an interface between a higher level control, that defines the synergy reference values $z$, and the robotic hand. The high level can be thought as independent from the robotic hand structure. The interface, based on the proposed mapping strategy, represents the low level control stage whereby the input synergies are translated into reference joint values which actually control the robotic hand.

This mapping has been tested in manipulation tasks. Work is in progress to validate the virtual ellipsoid mapping also for the approaching phase of grasps.

Simulation results are very interesting in terms of performances as shown in the previous section. However this approach presents some drawbacks.

The proposed mapping is based on a heuristic approach: we choose to reproduce a part of the hand motion, which practically corresponds to move and squeeze an ellipsoidal object. Although squeezing and moving an object explains a wide range of tasks, many other possibilities exist in 
manipulating objects which are not modelled with this mapping. Work is in progress to generalize the proposed method enriching the possible motions to be reproduced.

Differently from the joint to joint mapping, with respect to which the proposed method gets better performances, here $S_{r}$ is not a constant matrix but it depends on both the human and robotic hand configurations and by the reference position points of the human and robotic hands which should be given in this work.

\section{CONCLUSION AND FutURE WORK}

Designing synergy-based control strategies in the paradigmatic hand domain can dramatically reduce the dimensionality of the grasping and manipulation problems for robotic hands. However, an efficient mapping is needed to deal with robotic hands with dissimilar kinematics. We propose a method for mapping synergy matrices that using a virtual object allows to specify the mappings directly in the task space thus avoiding the problem of dissimilar kinematics between human-like hand and robotic hands. We compared our solution to the most used solutions existing in literature and we evinced that the proposed method is more efficient in terms of mapped grasp quality and direction of motion. Our preliminary results seem to be very promising, they were performed on a robotic three fingers 9 DoFs hand, a modular hand with a kinematic structure very different from that of the human hand, and also on a 15 DoFs DLR HIT II robotic hand. The results showed the effectiveness of the proposed method. Further investigation on different robotic hands have been already planned. One of the main issue of our approach is that the mapping is not linear and that its implementation could need a high computational burden. The ongoing research is evaluating the conditions whereby some simplification can be applied to get constant or slowly varying mapping. As future work, moreover, an integration with grasping simulator like Grasp-it! [26] is expected in order to use its grasp planner to determine initial position of the human and the robotic hand.

\section{REFERENCES}

[1] B. B. Edin, L. Ascari, L. Beccai, S. Roccella, J. J. Cabibihan, and M. Carrozza, "Bio-inspired sensorization of a biomechatronic robot hand for the grasp-and-lift task," Brain Research Bulletin, vol. 75, no. 6 , pp. $785-795,2008$.

[2] L. Zollo, S. Roccella, E. Guglielmelli, M. Carrozza, and P. Dario, "Biomechatronic design and control of an anthropomorphic artificial hand for prosthetic and robotic applications," Mechatronics, IEEE/ASME Transactions on, vol. 12, no. 4, pp. $418-429,2007$.

[3] J. F. Soechting and M. Flanders, "Flexibility and repeatability of finger movements during typing: Analysis of multiple degrees of freedom," Journal of Computational Neuroscience, vol. 4, pp. 29-46, 1997.

[4] M. Santello, M. Flanders, and J. Soechting, "Postural hand synergies for tool use," The Journal of Neuroscience, vol. 18, no. 23, pp. 10 10510115 , December 1998.

[5] G. Gioioso, G. Salvietti, M. Malvezzi, and D. Prattichizzo, "Mapping synergies from human to robotic hands with dissimilar kinematics: an object based approach," in IEEE ICRA 2011 Workshop on Manipulation Under Uncertainty, Shanghai, China, 2011.

[6] M. Fischer, P. Van der Smagt, and G. Hirzinger, "Learning techniques in a dataglove based telemanipulation system for the dlr hand," in Robotics and Automation, 1998. Proceedings. 1998 IEEE International Conference on, vol. 2, May 1998, pp. 1603-1608.
[7] H. Hu, J. Li, Z. Xie, B. Wang, B. Liu, and G. Hirzinger, "A robot arm/hand teleoperation system with telepresence and shared control," pp. 1312-1317, 2005

[8] S. Ekvall and D. Kragic, "Interactive grasp learning based on human demonstration," in Robotics and Automation, 2004. Proceedings. ICRA '04. 2004 IEEE International Conference on, vol. 4, May 2004, pp. 3519-3524

[9] J. Aleotti and S. Caselli, "Grasp recognition in virtual reality for robot pregrasp planning by demonstration," in Robotics and Automation, 2006. ICRA 2006. Proceedings 2006 IEEE International Conference on, 2006, pp. 2801-2806.

[10] M. T. Ciocarlie and P. K. Allen, "Hand posture subspaces for dexterous robotic grasping," The International Journal of Robotics Research, vol. 28, no. 7, pp. 851-867, July 2009.

[11] A. Peer, S. Einenkel, and M. Buss, "Multi-fingered telemanipulation - mapping of a human hand to a three finger gripper," in Robot and Human Interactive Communication, 2008. RO-MAN 2008. The 17th IEEE International Symposium on, August 2008, pp. 465-470.

[12] J. Liu and Y. Zhang, "Mapping human hand motion to dexterous robotic hand," 2007 IEEE International Conference on Robotics and Biomimetics (ROBIO), pp. 829-834, Dec. 2007.

[13] L. Pao and T. Speeter, "Transformation of human hand positions for robotic hand control," in Robotics and Automation, 1989. Proceedings., 1989 IEEE International Conference on. IEEE, 1989, pp. 1758-1763.

[14] P. Gorce and N. Rezzoug, "A method to learn hand grasping posture from noisy sensing information," Robotica, vol. 22, no. 03, pp. 309318, 2004.

[15] S. B. Kang and K. Ikeuchi, "Robot task programming by human demonstration: mapping human grasps to manipulator grasps," in Intelligent Robots and Systems '94. 'Advanced Robotic Systems and the Real World', IROS '94. Proceedings of the IEEE/RSJ/GI International Conference on, vol. 1, September 1994, pp. 97-104.

[16] W. Griffin, R. Findley, M. Turner, and M. Cutkosky, "Calibration and mapping of a human hand for dexterous telemanipulation," in ASME IMECE 2000 Symposium on Haptic Interfaces for Virtual Environments and Teleoperator Systems, 2000, pp. 1-8.

[17] H. Wang, K. Low, M. Wang, and F. Gong, "A mapping method for telemanipulation of the non-anthropomorphic robotic hands with initial experimental validation," in Robotics and Automation, 2005. ICRA 2005. Proceedings of the 2005 IEEE International Conference on, april 2005, pp. $4218-4223$.

[18] D. Prattichizzo, M. Malvezzi, and A. Bicchi, "On motion and force controllability of grasping hands with postural synergies," in Proceedings of Robotics: Science and Systems, Zaragoza, Spain, June 2010.

[19] R. Murray, Z. Li, and S. Sastry, A mathematical introduction to robotic manipulation. CRC, 1994.

[20] D. Prattichizzo and J. Trinkle, "Grasping," in Handbook on Robotics, B. Siciliano and O. Kathib, Eds. Springer, 2008, pp. 671-700.

[21] C. Brown and H. Asada, "Inter-finger coordination and postural synergies in robot hands via mechanical implementation of principal components analysis," in Intelligent Robots and Systems, 2007. IROS 2007. IEEE/RSJ International Conference on. IEEE, 2007, pp. 28772882.

[22] M. Ciocarlie, C. Goldfeder, and P. Allen, "Dimensionality reduction for hand-independent dexterous robotic grasping," in Intelligent Robots and Systems, 2007. IROS 2007. IEEE/RSJ International Conference on, 2007, pp. 3270-3275.

[23] M. Gabiccini, A. Bicchi, D. Prattichizzo, and M. Malvezzi, "On the role of hand synergies in the optimal choice of grasping forces," Autonomous Robots, Springer, vol. 31, pp. 235-252, 2011.

[24] H. Liu, K. Wu, P. Meusel, N. Seitz, G. Hirzinger, M. Jin, Y. Liu, S. Fan, T. Lan, and Z. Chen, "Multisensory five-finger dexterous hand: The dlr/hit hand ii," in Intelligent Robots and Systems, 2008. IROS 2008. IEEE/RSJ International Conference on, 2008, pp. 3692 -3697.

[25] A. Bicchi, "On the closure properties of robotic grasping," The Int. J. of Robotics Research, vol. 14, no. 4, pp. 319-334, 1995.

[26] A. Miller and P. Allen, "Graspit! a versatile simulator for robotic grasping," Robotics \& Automation Magazine, IEEE, vol. 11, no. 4, pp. 110-122, 2005. 